\title{
Ultrafast electron diffraction imaging of bond breaking in di-ionized acetylene
}

B. Wolter ${ }^{1, \dagger}$, M. G. Pullen, ${ }^{1, \dagger}$, A.-T. Le ${ }^{2}$, M. Baudisch ${ }^{1}$, K. Doblhoff-Dier ${ }^{3}$, A. Senftleben ${ }^{4}$, M. Hemmer ${ }^{1,5}$, C. D. Schröter ${ }^{6}$, J. Ullrich ${ }^{6,7}$, T. Pfeifer ${ }^{6}$, R. Moshammer ${ }^{6}$, S. Gräfe ${ }^{8,9}$, O. Vendrell ${ }^{5,10}$, C. D. Lin ${ }^{2}$, J. Biegert ${ }^{1,11, *}$ ${ }^{1}$ ICFO-Institut de Ciencies Fotoniques, The Barcelona Institute of Science and Technology, 08860 Castelldefels (Barcelona), Spain

2J. R. Macdonald Laboratory, Physics Department, Kansas State University, Manhattan, Kansas 66506-2604, USA

${ }^{3}$ Leiden Institute of Chemistry, Gorlaeus Laboratories, Leiden University, Post Office Box 9502, 2300 RA Leiden, Netherlands

${ }^{4}$ Universität Kassel, Institut für Physik und CINSaT, Heinrich-Plett-Str. 40, 34132 Kassel, Germany

${ }^{5}$ Center for Free-Electron Laser Science, DESY, Notkestrasse 85, D-22607 Hamburg, Germany

${ }^{6}$ Max-Planck-Institut für Kernphysik, Saupfercheckweg 1, 69117 Heidelberg, Germany

${ }^{7}$ Physikalisch-Technische Bundesanstalt (PTB), Bundesallee 100, 38116 Braunschweig, Germany

${ }^{8}$ Institute for Physical Chemistry, Friedrich-Schiller University Jena, 07743 Jena, Germany

${ }^{9}$ Abbe Center of Photonics, Friedrich-Schiller-University Jena, 07743 Jena, Germany

${ }^{10}$ The Hamburg Centre for Ultrafast Imaging (CUI), Luruper Chaussee 149, D-22761 Hamburg, Germany

${ }^{11}$ ICREA-Institució Catalana de Recerca i Estudis Avançats, 08010 Barcelona, Spain

${ }^{\dagger}$ Authors contributed equally to this work

*Correspondence to: jens.biegert@icfo.eu

\begin{abstract}
Visualizing chemical reactions as they occur requires atomic spatial and femtosecond temporal resolution. Here, we report imaging of the molecular structure of acetylene $9 \mathrm{fs}$ after ionization. Using mid-infrared laser induced electron diffraction (LIED) we obtain snapshots as a proton departs the $\left[\mathrm{C}_{2} \mathrm{H}_{2}\right]^{2+}$ ion. By introducing an additional laser field, we also demonstrate control over the ultrafast dissociation process and resolve different bond dynamics for molecules oriented parallel vs. perpendicular to the LIED field. These measurements are in excellent agreement with a quantum chemical description of fielddressed molecular dynamics.
\end{abstract}


One Sentence Summary: We demonstrate space and time imaging of a single acetylene molecule after $9 \mathrm{fs}$ while one of its bonds is broken and a proton departs the molecule.

Ultrafast imaging of atomic motion in real time during transitions in molecular structure is prerequisite to disentangling the complex interplay between reactants and products $(1,2)$ since the motion of all atoms are coupled. Ultrafast absorption and emission spectroscopic techniques have uncovered numerous insights in chemical reaction dynamics $(3,4)$, but are limited by their reliance on local chromophores and their associated ladders of quantum states rather than global structural characterization.

Reaction imaging at the molecular level requires a combination of few-femtosecond temporal and picometer spatial measurement resolution (5). Amongst the many techniques that are currently under intense development, $x$-ray scattering can reach few-femtosecond pulse durations at photon energies of $8.3 \mathrm{keV}(1.5 \AA)$ (6) with a demonstrated measurement resolution of $3.5 \AA$ (7). Challenges for such photonbased approaches are the coarse spatial resolution and the low scattering cross-sections, especially for gas phase investigations. Electron scattering (8) provides much larger interaction cross-sections and smaller de Broglie wavelengths, but suffers from space charge broadening which decreases the temporal resolution. Consequently, measurements have demonstrated $7 \mathrm{pm}$ spatial and $100 \mathrm{fs}$ temporal resolution $(9,10)$ in gas phase experiments. Remedies to improve temporal resolution include relativistic electron acceleration (11) or electron bunch compression (12) with $100 \mathrm{fs}$ and $28 \mathrm{fs}$ limits, respectively. Compared to such incoherent scattering of electrons from an electron source off a molecular target, laser induced electron diffraction (LIED) is a self-imaging method based on coherent electron scattering (13-17). In LIED, one electron which is liberated from the target molecule through tunnel ionization, it is then accelerated in the field and rescattered of its molecular ion thereby acquiring structural information. The electron recollision process occurs within one optical cycle of the laser field and permits mapping electron momenta to recollision time $(18,19)$.

Here, we used LIED to image an entire hydrocarbon molecule (acetylene $-\mathrm{C}_{2} \mathrm{H}_{2}$ ) at 9 fs after ionizationtriggered dissociation and visualize the departure of a proton. Our methodology combines mid-IR LIED with single molecule coincidence detection in a reaction microscope (20-22) and used an additional laser control 
field to impulsively align the molecule (see Supplementary Materials). The laser control field, a $1700 \mathrm{~nm}$ pulse, is sent prior to the $3100 \mathrm{~nm}$ LIED pulse and, orients the rotationally cold acetylene molecule parallel or perpendicular to the LIED field depending on the time delay. The $3100 \mathrm{~nm}$ pulse triggers molecular dissociation and, at the same time, collects structural snapshots of the entire acetylene molecule. The alignment dependent dynamics (23) are structurally imaged for both orientations We chose acetylene because it is one of the best studied hydrocarbons (24-27) and offers the numerous degrees of freedom and multitude of structural dynamics found also in larger and more complex molecules $(1,28,29)$. Note that due to the strong field nature of LIED, many different fragmentation channels and ionization states occur simultaneously. Our specific implementation of mid-IR LIED with single molecule coincidence detection provides however a remedy since post selection of data regains channel selectivity. Using this capability, we specifically chose to isolate and examine the dissociation of the acetylene dication $\left(\mathrm{C}_{2} \mathrm{H}_{2}{ }^{2+} \rightarrow \mathrm{H}^{+}+\mathrm{C}_{2} \mathrm{H}^{+}\right)$ since it results in a proton $\mathrm{H}^{+}$and an ethynyl $\mathrm{C}_{2} \mathrm{H}^{+}$moiety. This prototypical dissociation pathway is interesting as it presents one of the fastest expected proton motions and can proceed via two different pathways.

Figure $1 \mathrm{~A}$ depicts the relevant cuts through the potential energy surface (PES) of acetylene along one $\mathrm{C}-\mathrm{H}$ direction, including states which are relevant for the desired dissociation channel of $\mathrm{C}_{2} \mathrm{H}_{2}{ }^{2+}(30,31)$ at the present experimental conditions using a $65 \mathrm{fs}(6.3$-cycle), $3100 \mathrm{~nm}$ pulse $(32,33)$ with a peak intensity of $(65 \pm 16) \mathrm{TW} / \mathrm{cm}^{2}$. The pulse parameters were chosen to position the experiment in the sequential double ionization (SDI) regime in which the LIED imaging electron is ejected independently from the first electron in a second tunnel ionization step before scattering off the acetylene dication (see Supplementary Materials). It is known $(26,30,31)$ that the resulting pair of proton $\mathrm{H}^{+}$and ethynyl $\mathrm{C}_{2} \mathrm{H}^{+}$moiety originate via different possible pathways from the $\mathrm{C}_{2} \mathrm{H}_{2}{ }^{2+}$ dication: (i) the first dissociative excited singlet and triplet states, the ${ }^{3} \Pi_{u}$ and the ${ }^{1} \Pi_{u}$ states, can be reached by ionization from the $\sigma_{g}$ type HOMO-1 of the neutral ground state $\left(1 \sigma_{\mathrm{g}}{ }^{2}, 1 \sigma_{\mathrm{u}}{ }^{2}, 2 \sigma_{\mathrm{g}}{ }^{2}, 2 \sigma_{\mathrm{u}}{ }^{2}, 3 \sigma_{\mathrm{g}}{ }^{2}, 1{\pi_{\mathrm{u}}}^{2}, 1{\pi_{\mathrm{u}}}^{2}\right)$ followed by tunnel ionization from the $\pi_{\mathrm{u}}$ type HOMO of the excited cationic ${ }^{2} \Sigma_{\mathrm{g}}{ }^{+}$state $(26,34)$; (ii) the dication possesses a long lived metastable triplet state $\left({ }^{3} \Sigma_{\mathrm{g}}{ }^{\circ}\right.$ lifetime $108 \mathrm{~ns}(35))$ and two metastable singlet states $\left({ }^{1} \Sigma_{\mathrm{g}}{ }^{+}\right.$and $\left.{ }^{1} \Delta_{\mathrm{g}}\right)$ that can be reached via sequential tunnel ionization of two $\pi_{u}$ electrons. In this case, first one electron tunnels from the HOMO of the neutral 
ground state $\left({ }^{1} \Sigma_{g}^{+}\right)$, populating the singly ionic doublet ${ }^{2} \Pi_{u}$ state; then a second electron tunnels from the HOMO of the singly ionic ground state to populate one of the aforementioned dicationic states. We were interested in the direct dissociation channel that leads to fast proton loss. A simple estimation of the initial speed of the $\mathrm{C}-\mathrm{H}$ bond elongation, which is based on the curvature of the ${ }^{3} \Pi_{\mathrm{u}}$ PES, yields an initial elongation velocity of $9 \mathrm{pm} / \mathrm{fs}$ from the initial C-H bond length of $1.07 \AA$ in the Frank-Condon region.

To obtain the clearest possible conditions for imaging the direct proton loss channel, and to investigate its dependence on the LIED field, we impulsively aligned the acetylene molecule with an additional $1700 \mathrm{~nm}$, $98 \mathrm{fs}$ pulse focused to a peak intensity of $(20 \pm 5) \mathrm{TW} / \mathrm{cm}^{2}$ into the interaction region of the $3100 \mathrm{~nm}$ LIED pulse. Having ensured that the $1700 \mathrm{~nm}$ pulse did not induce ionization, we could distinguish between the different pathways from a photo-ion/photo-ion coincidence (PIPICO) analysis of our data, which is shown in Fig 1B (here for perpendicularly oriented $\mathrm{C}_{2} \mathrm{H}_{2}$ ). The diagonally sloping line (upper left to lower right) shows a very pronounced section (left upper corner - (i) centred around $0.8 \mu \mathrm{s} / 4.1 \mu \mathrm{s}$ ), which corresponds to direct dissociation of the dication from its exited ${ }^{1} \Pi_{u}$ and ${ }^{3} \Pi_{u}$ states. Section (ii) of this line (left upper corner $1.0 \mu \mathrm{s} / 4.0 \mu \mathrm{s}$ to right lower corner $2.6 \mu \mathrm{s} / 3.1 \mu \mathrm{s}$ ) is much weaker and is identified with the dication's meta-stable states ${ }^{3} \Sigma_{\mathrm{g}},{ }^{1} \Delta_{\mathrm{g}}$ and ${ }^{1} \Sigma_{\mathrm{g}}{ }^{+}(36)$. The proton-ethynyl pairs of the fast deprotonation channel are accompanied by two shallow lines, above and below, respectively associated with reactions involving a ${ }^{13} \mathrm{C}$ atom and a neutral product that is not detected by the reaction microscope: $\mathrm{C}_{2} \mathrm{H}_{2}{ }^{2+} \rightarrow \mathrm{H}^{+}+$ $\mathrm{C}_{2}{ }^{+}+\mathrm{H}$. Both of these processes, as well as the slow proton loss channel, occur with a probability two orders of magnitude lower than that for the fast proton loss channel. Nevertheless, we explicitly excluded those channels by isolating the three-dimensional momentum distribution of the electrons which correspond to the reaction moieties originating from the fast proton loss channel. This permits extraction of structural information only from electrons that rescattered off the $\mathrm{H}^{+}$and $\mathrm{C}_{2} \mathrm{H}^{+}$fragment pairs from direct dissociation. Additional support to identify population of the fast proton ejection channel (i) stems from measurements of the fragment pair ion yield $\left(\mathrm{H}^{+}+\mathrm{C}_{2} \mathrm{H}^{+}\right)$as a function of delay between alignment and LIED pulse. Figure $1 \mathrm{C}$ shows the results, which are in excellent agreement with previous work (37) in which this temporal dependence was used to identify the two different dication fragmentation channels. 
Having identified the direct proton ejection channel (via ${ }^{1} \Pi_{u}$ and ${ }^{3} \Pi_{u}$ ), we turned to extracting structural information from its scattered electrons. Our LIED methodology (20) permits using momentum coincidence arguments to associate the scattering electron to only the moieties of the fast proton loss channel. Applying such constraints to the data analysis reduces the event rate by a factor 83 , but the elimination of the general electron scattering background ensures that the resulting momentum map of the electrons bears only structural information of the dissociating acetylene dication (shown in Fig. 2A); the left half of the image shows data for parallel, the right half of the image for perpendicularly, oriented acetylene. These momentum maps correspond to the doubly differential electron scattering cross-section measured under the influence of the mid-IR LIED field. However, molecular structure is extracted from the field-free molecular differential cross section (mDCS), which is to say that we need to correct for the influence of the mid-IR field. Here, operating LIED with the $3100 \mathrm{~nm}$ pulse in the deep tunneling regime permits application of the semiclassical model $(18,38)$ to determine the vector potential of the LIED field at the time of rescattering for a given electron energy. The vector potential presents the LIED field's influence on the rescattering electron as an offset from zero (field-free) momentum that is simply subtracted from the measurement. The mDCS is then extracted by recording the number of counts along the circumference of a circle whose radius corresponds to the scattering momentum and whose origin is shifted by the vector potential from zero momentum; we show an example in black in Fig. 2A for an electron kinetic energy of $50 \mathrm{eV}$. Information about the position of the molecule's nuclei is encoded as energy modulations onto the mDCS due to scattering interference. To make these oscillations directly visible, we calculate the molecular contrast factor (MCF), a renormalized quantity, from the mDCS (see Supplementary Materials).

Figure $2 \mathrm{~B}$ shows the resulting MCF for both perpendicularly oriented (red circles) and parallel oriented (blue squares) acetylene. Striking differences are immediately apparent between the MCFs of both orientations, indicating that different structures are observed. The positions of the individual scattering centers, and hence atomic distances, are extracted by calculating MCF patterns for a wide range of possible positions. Comparing the measured MCF with these patterns, we obtain the full three dimensional solution space (minimum fitted $\chi^{2}$ value as a function of the $\mathrm{C}-\mathrm{C}$ distance and two $\mathrm{C}-\mathrm{H}$ distances) of the instant condition of the molecular wave packet at the time of the electron's return. Note that the solution 
space is obtained without assumptions such as partially frozen nuclei or linear elongation and includes independent symmetric as well as asymmetric elongation of $\mathrm{C}-\mathrm{H}$ bonds.

We obtain two different solutions, one for the parallel and one for the perpendicular case, and present 2D cuts through the 3D solution space at those positions: Figure $2 \mathrm{C}$ depicts the solution for the parallel case for which we measure a C-C bond length of $(1.48 \pm 0.11) \AA$, a value $23 \%$ greater than the $1.204 \AA$ equilibrium bond length(39) in neutral acetylene. Associated C-H distances are $(2.31 \pm 0.15) \AA$ and $(1.19 \pm$ 0.10) $\AA$ corresponding to $118 \%$ and $12 \%$ elongations relative to the $1.06 \AA$ equilibrium value (39). This dramatic difference, in which one proton has more than doubled its distance to its neighbour, is a clear signature of departure of a proton and hence bond cleavage. The scenario is markedly different for the perpendicular case, where we measure a C-C bond elongation of $16 \%$ from the equilibrium value to $(1.38 \pm$ $0.06) \AA$. Figure $2 \mathrm{D}$ shows the measured $\mathrm{C}-\mathrm{H}$ distances of $(1.94 \pm 0.10) \AA$ and $(1.54 \pm 0.06) \AA$. The more symmetric scenario of $\mathrm{C}-\mathrm{H}$ bond elongations by $83 \%$ and $45 \%$ from their equilibrium value is understandable considering the molecule is aligned perpendicular to the control field when being imaged by the LIED electrons; i.e. the molecule is not asymmetrically pulled apart by the strong laser field. Figure 3 shows that this approximates an imaging scenario under quasi field-free conditions. The measured disparity of C$\mathrm{H}$ distances for different alignment provides a means of controlling bond cleavage and proton loss depending on molecular orientation.

Next we sought to explain the difference in imaged structures between the parallel and perpendicularly aligned molecules. We turn to mixed quantum chemistry and semi-classical ab-initio molecular dynamics calculations to realistically describe the molecular wave packet in the dressing mid-IR field with varying polarization direction as a function of time; see Supplementary Materials for details. Figure 3 shows how the PESs of the dication are modified for parallel (Fig. 3A) and perpendicularly oriented (Fig. 3B) molecules in the presence of the LIED field. Both figures show, for clarity, only the dication's ground states $\left({ }^{3} \sum_{\mathrm{g}}{ }^{-}\right.$and the near degenerate singlet states $\left.{ }^{1} \Delta_{g},{ }^{1} \Sigma_{g}^{+}\right)$and the ${ }^{1} \Pi_{u}$ and ${ }^{3} \Pi_{u}$ excited states. For the parallel case (Fig. $3 A$ ), we find a stable equilibrium when the LIED field is directed so as to pull the hydrogen atom towards the carbon atom (green). Once the LIED field direction reverses, half a cycle later, all PESs (blue) become strongly dissociative and the $\mathrm{C}-\mathrm{H}$ bond is broken within $8 \mathrm{fs}$. Figure 3 shows internuclear separation for only 
one of the two $\mathrm{C}-\mathrm{H}$ bonds, i.e. the exact opposite scenario occurs at the same time for the other $\mathrm{C}-\mathrm{H}$ bond (exchange blue with green PESs). In total, during one LIED field cycle (10.3 fs for $3100 \mathrm{~nm}$ ), one $\mathrm{C}-\mathrm{H}$ is always broken while the other $\mathrm{C}-\mathrm{H}$ bond is only elongated. We find excellent agreement with our measured C-C bond length, which is elongated during both half-cycles of the LIED field. The calculation yields a C-C bond length of $1.45 \AA$ in excellent agreement with our measured value of $(1.48 \pm 0.11) \AA$. The perpendicular case is different as no preferential axis is induced by the LIED field and hence there are two degenerate cases. Thus, Fig. 3B exhibits no LIED field direction dependence and only minimal modification of the field free PESs. This scenario leads to slower bond dynamics with eventual breakup. The computed smaller C-C elongation to $1.40 \AA$ agrees with the measured bond length of $(1.38 \pm 0.06) \AA$. The simulations show that the $\mathrm{C}-\mathrm{C}$ bond and the second $\mathrm{C}-\mathrm{H}$ bond undergo vibration throughout the course of the LIED pulse, yet they stay bound within the temporal range of one optical cycle $(10.3 \mathrm{fs})$.

Because the LIED field does not noticeably distort the PESs in the perpendicular orientation, the acetylene dication behaves like a quasi--field-free electronic system and can be imaged as such. Moreover, the dependence of proton loss dynamics on alignment permits control over the speed and visualization of molecular dissociation.

Having corroborated the dependence of proton loss on alignment, we next linked the measured structures to the times when the snapshots were taken. In our experiment, we analyzed electrons with $50 \mathrm{eV}$ return energy because they yielded the highest number of counts for the largest angular coverage and hence the best scattering momentum transfer. Based on the semiclassical model $(18,38)$, which pertains well for our Keldysh parameter of $\gamma=0.31$, we can determine the time of return and the backscattering energy for the scattering electron. We only have to consider the long trajectory pathway due to its much higher ionization probability compared with the short trajectory. We show in Fig. 4A that these electrons have reencountered the target after $9.15 \mathrm{fs}(10.3 \mathrm{fs}$ correspond to 1 optical cycle at $3100 \mathrm{~nm})$ and backscatter with maximum energy of $188 \mathrm{eV}$. We additionally analyzed our measurement for two closely neighboring energies of 48 and $52 \mathrm{eV}$, which correspond to backscattering energies of 181 and $195 \mathrm{eV}$, and for which we achieve high count rates with excellent signal to noise. The overall temporal spread is negligible because 
these three measurements interrogated the molecular structure during a short span between 9.1 and 9.2 fs, indicated by the vertical grey bars in Fig. 4. The experimental results for the three scattering energies (circles with error bars) are overlaid with calculated values for all bond distances and for all orientations. The calculated values are based on modeling the experimental conditions with ab initio molecular dynamics simulations that take the modification of the dication states of acetylene by the LIED field fully into account. Figures 4B-G show the simulated evolution of the corresponding probability distribution on the dissociative excited dication state over a time span of $20 \mathrm{fs}$. For parallel orientation (Figs. 4B,D,F), the molecule experiences the full LIED field strength along its molecular axis and gets maximally distorted. This scenario corresponds to rapid elongation of one $\mathrm{C}-\mathrm{H}$ bond (Fig. 4B) and breakage, defined at twice its equilibrium distance, after $8 \mathrm{fs}$. The other C-H bond (Fig. 4D) experiences elongation in the presence of the LIED field and the C-C bond (Fig. 4F) moves with a period significantly longer than the LIED field's optical cycle. The dynamics change markedly for perpendicular orientation (see Figs. 4C,E,F), which closely approximates a quasi-field-free imaging scenario (see Fig. 3). Here, we find that the C-C bond undergoes very similar excursion as for the parallel case due to the stiffness of the bond. The two $\mathrm{C}-\mathrm{H}$ bonds however show strong probabilities to both oscillate in phase with the LIED field with some small probability for dissociation. This behavior make sense, over the shown time range, as there is no preferential direction of the external field that would bias the dynamics of one $\mathrm{C}-\mathrm{H}$ bond compared with the other.

The measured snapshots are overlaid (circles) with all figures for the parallel case (Fig. 4B,D,F) and exhibit excellent agreement with the expected behavior of the molecule. The result from Fig. $2 \mathrm{C}$ is indicated by the pink filled circle. Similarly, we show the perpendicular case in Figs. 4C,E,G and find equally excellent agreement with the measurement shown in Fig. 2D.

Based on these findings we can corroborate the full spatio-temporal structure of dicationic acetylene $9 \mathrm{fs}$ after ionization. The snapshots of the spatio-temporal structure were taken with an estimated $0.6 \mathrm{fs}$ temporal resolution and are capable of distinguishing the different kinetic behaviors of the molecule when field-ionized parallel or perpendicular to the LIED field. In the parallel case, the snapshots show that one of the hydrocarbon bonds is heterolytically cleaved with the proton $1.24 \AA$ away from its equilibrium 
position. The perpendicular case snapshots reveal the molecular structure in the quasi--field-free scenario for the dissociative dication.

As a future step, we envision application of our implementation of LIED to triggering and imaging of ultrafast structural transformations over a longer timescale, e.g. with two separate pulses as pump and probe, and with molecules with more complex structures. Prospects include structural and spatial isomerization and especially proton tautomerization, a key chemical and biological process that is largely obscured from $x$-ray scattering techniques.

\section{Acknowledgements}

We acknowledge financial support from the Spanish Ministry of Economy and Competitiveness (MINECO), through the "Severo Ochoa" Programme for Centres of Excellence in R\&D (SEV-2015-0522), FIS2014-56774R, FIS2014-51478-ERC, the Catalan Institució Catalana de Recerca I Estudis Avançats, Agencia de Gestió d'Ajuts Universitaris i de Recerca (AGAUR) with SGR 2014-2016, the Fundació Cellex Barcelona, the European Union's Horizon 2020 research and innovation programme under LASERLAB-EUROPE (EU-H2020 654148), COST Actions MP1203, XLIC, the Marie Sklodowska-Curie grant agreement No. 641272 and the European Research Council through ERC-2013 Advanced Grant No. 338580. B.W. was supported by AGAUR (FI-DGR 2013-2015). M.G.P. was supported by the ICFONEST+ program, partially funded by the Marie Curie co-funding of Regional, National and International Programs-COFUND (FP7-PEOPLE-2013-COFUND) action of the European Commission. A.-T.L. and C.D.L. are supported by the Chemical Sciences, Geosciences, and Biosciences Division, Office of Basic Energy Sciences, Office of Science, U.S. Department of Energy (DOE) under Grant No. DE-FG02-86ER13491. We thank Dr. Dobryna Zalvidea, Dr. Michele Sclafani and Prof. Dr. Albert Stolow for helpful and inspiring discussions.

Fig. 1. Identification of the relevant states leading to proton ejection. (A) shows the calculated relevant energy levels and possible pathways (34) leading to dissociation of acetylene and departure of one proton 
(see e.g. Refs. $(26,35)$, the calculation is detailed in the Supplementary Materials). Two main pathways are identified, one leading to dissociative PES and fast dissociation (i), the other one via metastable states to slow dissociation (ii). These pathways are identified in the PIPICO analysis (B) and exhibit alignment dependent fragment yields $(C)$. (B) is shown here for perpendicularly oriented acetylene; for the parallel case see Figure S4.

Fig. 2. Measurement of bond distances with LIED. (A) shows the electron momentum distribution in coincidence with the moieties corresponding to the fast proton loss channel. The left half of the image shows data for parallel aligned, the right half for perpendicularly aligned, acetylene. The molecular contrast factor (MCF) is shown for both cases, parallel (top, blue) and perpendicular (bottom, red) in (B); error bars are derived from the experiment based on Poissonian statistics (see Supplementary Materials for details). (C) and (D) show experimental data (white data points with error bars extracted from the MCF fit) overlaid with numerical results (density plot) for cuts through the 3D solution space at the location of its minima. The locations of these cuts correspond to C-C distances of $1.48 \AA$ for the parallel case (C) and $1.37 \AA$ for the perpendicular case (D). (C) shows elongation of one $C-H$ bond to more than twice the equilibrium bond length, corresponding to bond breakage, while the other $\mathrm{C}-\mathrm{H}$ bond is still bound. (D) represents a more symmetric scenario nearing heterolytic cleavage of one of the two $\mathrm{C}-\mathrm{H}$ bonds.

Fig. 3. PES of the acetylene dication with the mid-IR LIED field. The field-free case is shown in (A) and (B) in red. (A) shows how the PESs Stark-shift when dressed by a mid-IR LIED field aligned with the molecular axis. For one field direction (E>0), all dication PESs are strongly dissociative (blue) whereas for the other field direction $(E<0)$, the PESs present a bound scenario. The trend is exactly reversed for the other $\mathrm{C}-\mathrm{H}$ bond. (B) shows the doubly degenerate scenario for alignment perpendicular to the mid-IR LIED field, for which we find slight PESs shifts, but not the dramatic Stark shifts shown in (A).

Fig. 4. Temporal dynamics of acetylene resolved for the different bonds and as function of alignment. (A) Temporal range imaged for electron energies ranging up to $195 \mathrm{eV}$ in accordance with the semiclassical 
rescattering model. Mixed quantum-classical dynamical calculations are shown below for $(B, D, F)$ parallel and $(C, E, G)$ perpendicularly aligned acetylene. Extracted bond distances from the snapshots taken between 9.1 to $9.2 \mathrm{fs}$ are overlaid onto the calculations and exhibit excellent agreement by being well positioned within the theoretical distribution; the results corresponding to Fig. 2 are shown in pink and error bars are determined based on the semiclassical rescattering model (see Supplementary Materials for details). Differences for bond elongation between the $(B, D)$ parallel and $(C, E)$ perpendicular cases are clearly resolved.

Supplementary Materials

Supplementary Text

Figs. S1 to S5

References (40-71) 


\section{References}

1. R. G. Woolley, Quantum chemistry beyond the Born-Oppenheimer approximation. J. Mol. Struct. THEOCHEM. 230, 17-46 (1991).

2. A. H. Zewail, Femtochemistry: Atomic-Scale Dynamics of the Chemical Bond †. J. Phys. Chem. A. 104, 5660-5694 (2000).

3. H. Xu, T. Okino, K. Yamanouchi, Tracing ultrafast hydrogen migration in allene in intense laser fields by triple-ion coincidence momentum imaging. J. Chem. Phys. 131, $151102(2009)$.

4. J. C. Polanyi, A. H. Zewail, Direct Observation of the Transition State. Acc. Chem. Res. 28, 119-132 (1995).

5. M. Chergui, A. H. Zewail, Electron and X-ray methods of ultrafast structural dynamics: advances and applications. Chemphyschem. 10, 28-43 (2009).

6. M. P. Minitti et al., Imaging Molecular Motion: Femtosecond X-Ray Scattering of an Electrocyclic Chemical Reaction. Phys. Rev. Lett. 114, 255501 (2015).

7. K. Ayyer et al., Macromolecular diffractive imaging using imperfect crystals. Nature. 530, 202-206 (2016).

8. S. A. Ischenko, A A; Aseyev, Ed., Time-Resolved Electron Diffraction - For Chemistry, Biology and Materials Science (Elsevier, 2014; http://www.sciencedirect.com/science/article/pii/B9780128001455210010), vol. 184 of Advances in Imaging and Electron Physics.

9. P. Zhang, J. Yang, M. Centurion, Tilted femtosecond pulses for velocity matching in gasphase ultrafast electron diffraction. New J. Phys. 16, 83008 (2014).

10. C. Gerbig, A. Senftleben, S. Morgenstern, C. Sarpe, T. Baumert, Spatio-temporal resolution studies on a highly compact ultrafast electron diffractometer. New J. Phys. 17, 43050 (2015).

11. S. Manz et al., Mapping atomic motions with ultrabright electrons: towards fundamental limits in space-time resolution. Faraday Discuss. 177, 467-91 (2015). 
12. A. Gliserin, M. Walbran, F. Krausz, P. Baum, Sub-phonon-period compression of electron pulses for atomic diffraction. Nat. Commun. 6, 8723 (2015).

13. T. Zuo, A. D. Bandrauk, P. B. Corkum, Laser-induced electron diffraction: a new tool for probing ultrafast molecular dynamics. Chem. Phys. Lett. 259, 313-320 (1996).

14. M. Lein, J. P. Marangos, P. L. Knight, Electron diffraction in above-threshold ionization of molecules. Phys. Rev. A. 66, 51404 (2002).

15. M. Meckel et al., Laser-induced electron tunneling and diffraction. Science (80-. ). 320, 1478-82 (2008).

16. C. D. Lin, A.-T. Le, Z. Chen, T. Morishita, R. Lucchese, Strong-field rescattering physics-self-imaging of a molecule by its own electrons. J. Phys. B At. Mol. Opt. Phys. 43, 122001 (2010).

17. C. I. Blaga et al., Imaging ultrafast molecular dynamics with laser-induced electron diffraction. Nature 483, 194-7 (2012).

18. P. B. Corkum, Plasma perspective on strong field multiphoton ionization. Phys. Rev. Lett. 71, 1994-1997 (1993).

19. S. Baker et al., Probing proton dynamics in molecules on an attosecond time scale. Science 312, 424-7 (2006).

20. M. G. M. G. Pullen et al., Imaging an aligned polyatomic molecule with laser-induced electron diffraction. Nat. Commun. 6, 7262 (2015).

21. B. Wolter et al., Strong-Field Physics with Mid-IR Fields. Phys. Rev. X. 5, 21034 (2015).

22. M. G. Pullen et al., Influence of orbital symmetry on diffraction imaging with rescattering electron wave packets. Nat. Commun. 7, 11922 (2016).

23. P. M. Kraus et al., Measurement and laser control of attosecond charge migration in ionized iodoacetylene. Science (80-. ). 350, 790-5 (2015).

24. X. Xie et al., Attosecond-recollision-controlled selective fragmentation of polyatomic molecules. Phys. Rev. Lett. 109, 243001 (2012).

25. A. S. Alnaser et al., Subfemtosecond steering of hydrocarbon deprotonation through superposition of vibrational modes. Nat. Commun. 5, 3800 (2014). 
26. B. Gaire et al., Photo-double-ionization of ethylene and acetylene near threshold. Phys. Rev. A. 89, 13403 (2014).

27. H. Ibrahim et al., Tabletop imaging of structural evolutions in chemical reactions demonstrated for the acetylene cation. Nat. Commun. 5, 4422 (2014).

28. G. A. Worth, L. S. Cederbaum, Beyond Born-Oppenheimer: molecular dynamics through a conical intersection. Annu. Rev. Phys. Chem. 55, 127-58 (2004).

29. J. M. Bowman et al., Chemistry. Beyond Born-Oppenheimer. Science. 319, 40-1 (2008).

30. E. M.-L. Ohrendorf, F. Tarantelli, L. S. Cederbaum, Dicationic states of hydrocarbons and a statistical approach to their Auger spectra. J. Chem. Phys. 92, 2984 (1990).

31. R. Thissen et al., Dissociations of the ethyne dication $\mathrm{C} 2 \mathrm{H} 2+2$. J. Chem. Phys. 99, 6590 (1993).

32. J. Biegert, P. K. P. K. Bates, O. Chalus, New Mid-Infrared Light Sources. IEEE J. Sel. Top. Quantum Electron. 18, 531-540 (2012).

33. M. Baudisch et al., Sub-4-optical-cycle, $340 \mathrm{MW}$ peak power, high stability mid-IR source at $160 \mathrm{kHz}$. J. Opt. 17, 94002 (2015).

34. K. Doblhoff-Dier, M. Kitzler, S. Gräfe, Theoretical investigation of alignment-dependent intense-field fragmentation of acetylene. Phys. Rev. A. 94, 13405 (2016).

35. M. Alagia et al., Angular and energy distribution of fragment ions in dissociative double photoionization of acetylene molecules at $39 \mathrm{eV}$. J. Chem. Phys. 136, 204302 (2012).

36. M. Alagia et al., Dissociative double photoionization of $\mathrm{CO} 2$ molecules in the $36-49 \mathrm{eV}$ energy range: angular and energy distribution of ion products. Phys. Chem. Chem. Phys. 12, 5389-95 (2010).

37. X. Xie et al., Selective control over fragmentation reactions in polyatomic molecules using impulsive laser alignment. Phys. Rev. Lett. 112, 163003 (2014).

38. J. Krause, K. Schafer, K. Kulander, High-order harmonic generation from atoms and ions in the high intensity regime. Phys. Rev. Lett. 68, 3535-3538 (1992).

39. CRC Handbook of Chemistry and Physics, 94th Edition - CRC Press Book, (available at https://www.crcpress.com/CRC-Handbook-of-Chemistry-and-Physics-94th- 
Edition/Haynes/9781466571143).

40. J. Biegert, P. K. P. K. Bates, O. Chalus, New Mid-Infrared Light Sources. IEEE J. Sel. Top. Quantum Electron. 18, 531-540 (2012).

41. M. Baudisch et al., Sub-4-optical-cycle, $340 \mathrm{MW}$ peak power, high stability mid-IR source at $160 \mathrm{kHz}$. J. Opt. 17, 94002 (2015).

42. M. G. Pullen et al., Imaging an aligned polyatomic molecule with laser-induced electron diffraction. Nat. Commun. 6, 7262 (2015).

43. B. Wolter et al., Strong-Field Physics with Mid-IR Fields. Phys. Rev. X. 5, 21034 (2015).

44. D. Shafir et al., Resolving the time when an electron exits a tunnelling barrier. Nature 485, 343 (2012).

45. S. M. Hankin, D. M. Villeneuve, P. B. Corkum, D. M. Rayner, Intense-field laser ionization rates in atoms and molecules. Phys. Rev. A. 64, 13405 (2001).

46. B. Walker et al., Precision measurement of strong field double ionization of helium. Phys. Rev. Lett. 73, 1227-1230 (1994).

47. X. Xie et al., Selective control over fragmentation reactions in polyatomic molecules using impulsive laser alignment. Phys. Rev. Lett. 112, 163003 (2014).

48. B. K. McFarland et al., High Harmonic Generation from Multiple Orbitals in $\mathrm{N}_{2}$. Science $322,1232(2008)$.

49. J. Ortigoso, M. Rodríguez, M. Gupta, B. Friedrich, Time evolution of pendular states created by the interaction of molecular polarizability with a pulsed nonresonant laser field. J. Chem. Phys. 110, 3870 (1999).

50. M. Alagia et al., Angular and energy distribution of fragment ions in dissociative double photoionization of acetylene molecules at $39 \mathrm{eV}$. J. Chem. Phys. 136, 204302 (2012).

51. B. Gaire et al., Photo-double-ionization of ethylene and acetylene near threshold. Phys. Rev. A. 89, 13403 (2014).

52. M. Head-Gordon, J. A. Pople, M. J. Frisch, MP2 energy evaluation by direct methods. Chem. Phys. Lett. 153, 503-506 (1988).

53. R. Krishnan, J. S. Binkley, R. Seeger, J. A. Pople, Self-consistent molecular orbital 
methods. XX. A basis set for correlated wave functions. J. Chem. Phys. 72, 650 (1980).

54. M. Frisch et al., Gaussian 09, Revision B.01. Gaussian 09, Revis. B.01, Gaussian, Inc., Wallingford CT (2009).

55. J. P. Finley, Similarities between single reference perturbation theory based on a CASSCF wavefunction and multireference perturbation theory based on a reference space spanned by a CAS. Chem. Phys. Lett. 283, 277-282 (1998).

56. K. P. Lawley, B. O. Roos, Advances in Chemical Physics (John Wiley \& Sons, Inc., Hoboken, NJ, USA, 1987; http://doi.wiley.com/10.1002/9780470142943), vol. 69 of Advances in Chemical Physics.

57. F. Aquilante et al., MOLCAS 7: the next generation. J. Comput. Chem. 31, 224-47 (2010).

58. V. Veryazov, P.-O. Widmark, L. Serrano-Andrés, R. Lindh, B. O. Roos, 2MOLCAS as a development platform for quantum chemistry software. Int. J. Quantum Chem. 100, 626-635 (2004).

59. G. Karlström et al., MOLCAS: a program package for computational chemistry. Comput. Mater. Sci. 28, 222-239 (2003).

60. F. Aquilante, P.-Å. Malmqvist, T. B. Pedersen, A. Ghosh, B. O. Roos, Cholesky Decomposition-Based Multiconfiguration Second-Order Perturbation Theory (CDCASPT2): Application to the Spin-State Energetics of Co(III)(diiminato)(NPh). J. Chem. Theory Comput. 4, 694-702 (2008).

61. M. . Feit, J. . Fleck, A. Steiger, Solution of the Schrödinger equation by a spectral method. J. Comput. Phys. 47, 412-433 (1982).

62. T. H. Dunning, Gaussian basis sets for use in correlated molecular calculations. I. The atoms boron through neon and hydrogen. J. Chem. Phys. 90, 1007 (1989).

63. M. W. Schmidt et al., General atomic and molecular electronic structure system. J. Comput. Chem. 14, 1347-1363 (1993).

64. R. Thissen et al., Dissociations of the ethyne dication $\mathrm{C} 2 \mathrm{H} 2+2$. J. Chem. Phys. 99, 6590 (1993). 
65. A. Kivimäki et al., Angle-resolved Auger spectra of the molecule. J. Phys. B At. Mol. Opt. Phys. 30, 4279-4291 (1997).

66. E. M.-L. Ohrendorf, F. Tarantelli, L. S. Cederbaum, Dicationic states of hydrocarbons and a statistical approach to their Auger spectra. J. Chem. Phys. 92, 2984 (1990).

67. J. C. Tully, Molecular dynamics with electronic transitions. J. Chem. Phys. 93, 1061 (1990).

68. I. Hargittai, M. Hargittai, Stereochemical Applications of Gas-phase Electron Diffraction: Structural information for selected classes of compounds (Vch Pub (November 1988), 1988 https://books.google.es/books/about/Stereochemical_Applications_of_Gas_phase.html? id=prgiAQAAIAAJ\&pgis=1).

69. Z. Chen, A.-T. Le, T. Morishita, C. D. Lin, Quantitative rescattering theory for laserinduced high-energy plateau photoelectron spectra. Phys. Rev. A. 79, 33409 (2009).

70. T. Morishita et al., Retrieval of experimental differential electron-ion elastic scattering cross sections from high-energy ATI spectra of rare gas atoms by infrared lasers. J. Phys. B At. Mol. Opt. Phys. 42, 105205 (2009).

71. A.-T. Le, R. R. Lucchese, S. Tonzani, T. Morishita, C. D. Lin, Quantitative rescattering theory for high-order harmonic generation from molecules. Phys. Rev. A. 80, 13401 (2009).

72. J. Xu, Z. Chen, A.-T. Le, C. D. Lin, Self-imaging of molecules from diffraction spectra by laser-induced rescattering electrons. Phys. Rev. A. 82, 33403 (2010) 